\title{
Humoral immunogenicity of ChAd63_MVA ME- TRAP vaccination in African infants and children
}

\author{
Georgina Bowyer ${ }^{1 *}$, Muhammed O Afolabi ${ }^{2}$, Carly M Bliss ${ }^{1}$, Alfred B Tiono ${ }^{3}$, Abdoulie Drammeh², Issa Nébié ${ }^{3}$, \\ Ya Jankey Jagne ${ }^{2}$, Jean Baptiste Yaro ${ }^{3}$, Egeruan B Imoukhuede ${ }^{4}$, Katie L Flanagan ${ }^{2}$, Beate Kampman², \\ Nicola Viebig ${ }^{5}$, Sodiomon B Sirima ${ }^{3}$, Kalifa Bojang ${ }^{2}$, Adrian VS Hill ${ }^{1,4}$, Katie J Ewer ${ }^{1}$
}

From Challanges in malaria research: Core science and innovation

Oxford, UK. 22-24 September 2014

\section{Background}

The only malaria vaccine to have been tested in Phase III clinical vaccine trials [1] induces protection which has been associated with high titres of antibodies against sporozoites. Efficacy against clinical malaria in infants of 5-17 months and 6-12 weeks was 55.8\% and $31.3 \%$ respectively. A successful malaria vaccine will likely need to induce both cellular and humoral immunity in order to achieve a deployable level of efficacy in the target age groups. Prime-boost immunisation with viral vectors ChAd63 and MVA, both encoding ME-TRAP, has been shown to induce high $\mathrm{T}$ cell responses and modest antibody responses to the pre-erythrocytic malaria antigen TRAP. A Phase II study with this vaccine regime in malaria-naïve adults showed significant efficacy, which correlated with frequency of monofunctional CD8+ T cells secreting IFN $\gamma$. TRAP antibody titres were modest and did not correlate with protection [2]. In contrast to this, high titres of vaccine-induced anti-TRAP antibodies are measured in infants in malaria-endemic settings.

\section{Materials and methods}

Antibody titres were measured in 138 malaria-exposed children vaccinated with ChAd63 MVA ME-TRAP in three Phase I studies in The Gambia and Burkina Faso. Age groups at first immunisation were 2-6 years, 5-12 months and 10 weeks in The Gambia and 5-17 months in Burkina Faso. Avidity and isotype profiles were also analyzed.

\section{Results}

Antibody responses to TRAP were significantly higher in 10 week old and 5-12 month old infants in The Gambia

Jenner Institute Laboratories, University of Oxford, Oxford, UK Full list of author information is available at the end of the article and 5-17 month old infants in Burkina Faso compared to 2-6 year old children and adults in The Gambia and malaria-naïve UK adults. IgG isotype responses were predominantly IgG1 and IgG3 and we also detected IgA and IgM. TRAP-specific IgG avidity was significantly higher in Burkinabe infants aged 5-17 months and Gambian infants aged 5-12 months compared to Gambian adults and 2-6 year old children. TRAP-specific IgG1 avidity significantly correlated with age at vaccination in 5-17 month old Burkinabe infants and was significantly higher than in 10 week old Gambian infants. Functional activity of anti-TRAP antibodies will be analysed in vitro using a sporozoite invasion inhibition assay.

\section{Conclusions}

We demonstrate excellent humoral immunogenicity in key target populations vaccinated with a pre-erythrocytic malaria vaccination regime, exceeding that seen in UK malaria-naïve adults.

\begin{abstract}
Authors' details
${ }^{1} J$ enner Institute Laboratories, University of Oxford, Oxford, UK. ${ }^{2}$ Medical Research Council Unit, Fajara, Gambia. ${ }^{3}$ Centre National de Recherche et de Formation sur le Paludisme, Ouagadougou, Burkina Faso. ${ }^{4}$ Centre for Clinical Vaccinology and Tropical Medicine, Oxford, UK. ${ }^{5}$ European Vaccine Initiative, Heidelberg, Germany.
\end{abstract}

Published: 22 September 2014

References

1. Vansadia P, et al: A Phase 3 Trial of RTS,S/AS01 Malaria Vaccine in African Infants. N Engl J Med 2012, 367:2284-2295.

2. Ewer $\mathrm{K}, \mathrm{O}^{\prime}$ Hara G: Protective CD8+ T-cell immunity to human malaria induced by chimpanzee adenovirus-MVA immunisation. Nat Commun 2013, 4.

doi:10.1186/1475-2875-13-S1-P16

Cite this article as: Bowyer et al:: Humoral immunogenicity of ChAd63_MVA ME-TRAP vaccination in African infants and children. Malaria Journal 2014 13(Suppl 1):P16. 